\title{
PRODUCCIÓN ESCRITA Y LITERACIDAD FAMILIAR ${ }^{1}$
}

\author{
Margarita Calderón López*
}

\section{Resumen}

El objetivo de esta investigación es indagar en la manera en la que, estudiantes de entre 7 y 10 años, se apropian de las prácticas y experiencias de lectura y escritura en el hogar en su producción textual. Específicamente, este estudio se enfoca en la construcción de significado mediante estrategias discursivas y la motivación hacia la escritura y la lectura basada en las prácticas de literacidad familiar (Cassany 2006). Para ello, 107 estudiantes completaron un cuestionario con preguntas de selección múltiple y una pregunta abierta de escritura. Los resultados muestran que la variable de género tiene un mayor impacto que la de edad en cuanto a la riqueza de las estrategias de construcción de significado. Por otro lado, el estudio reveló que las prácticas y creencias en torno a la lectura y escritura en el hogar no son estadísticamente significativas respecto de los resultados en la producción textual.

Palabras clave: Texto, construcción del conocimiento, literacidad.

\begin{abstract}
The aim of this study was to investigate the way in which students between 7 and 10 years, appropriate the practices and experiences of reading and writing at home in textual production. Specifically, this study focuses on meaning construction through discursive strategies and motivation towards reading and writing at home (Cassany 2006). For this, 107 students completed a questionnaire with multiple choice questions and an elicitation task for writing. The results showed that gender has a greater impact than age in terms of meaning making strategies. Furthermore, the study revealed that practices and beliefs about reading and writing at home are not statistically significant for results in textual production.
\end{abstract}

Key words: Text, meaning construction, literacy, literacidad.

Recibido: 30-07-2014

Aceptado: 05-05-2015

1 Este estudio forma parte de la tesis para obtener el grado de $\mathrm{PhD}$ en Lingüística (Lancaster University), parte de esta investigación fue presentada como ponencia en el 7th International Postgraduate Conference in Linguistics and Language Teaching, Lancaster University (13/07/2012), y en la VII conferencia ALED, Universidad de la Serena (17-19/10/2012). Algunos de los temas que fueron desarrollados en las conferencias no han sido tratados en este artículo.

* Nacionalidad chilena , Phd c en Lingüística , Lancaster University, m.caldern@lancaster.ac.uk 


\section{Contexto}

En los últimos años la preocupación por la producción escrita de los estudiantes se ha visto incrementada debido a intereses de índole académico, político y social. En este contexto, diversas investigaciones se han llevado a cabo lo que ha generado un amplio campo de conocimiento que ha contribuido en diversas formas a la mejora de la producción textual de los estudiantes(Crespo, Benítez et al. 2007; Figari 2009; Concha and Paratore 2011; Sotomayor, Molina et al. 2013). Sin embargo, aún existe poca investigación en relación a las percepciones sobre literacidad y cómo estas se relacionan con la construcción del significado en el texto escrito. Este estudio describe las creencias y prácticas de literacidad de niños de entre siete y 10 años para luego relacionarlas a la manera en que construyen un texto. Para ello, 107 alumnos de entre 7 y 10 años fueron invitados a responder una actividad que contemplaba un set de preguntas de selección múltiple y una pregunta de elicitación de escritura. Las preguntas de selección múltiple buscaban identificar percepciones y creencias de los estudiantes en torno de las prácticas de lectura y escritura. La pregunta abierta buscaba elicitar la escritura por medio de una pregunta que permitía a los estudiantes reconstruir una parte de su mundo (Barton 1994).

Por medio del análisis de la relación entre el texto escrito y las percepciones de los estudiantes respecto de la literacidad se espera demostrar que los participantes, aun a su corta edad, se relacionan como agentes de su producción textual y por ende, sus percepciones son relevantes a la hora de indagar en posibles estrategias para mejorar su producción textual. Esta distinción supone que los estudiantes son parte activa de su propio aprendizaje(Rogoff 1990) y en este sentido, se expone la relevancia de considerar sus percepciones en el estudio de su escritura. En este sentido, esta investigación supone una perspectiva social respecto de la lectura y la escritura, lo que expone la importancia de relevar ambos conceptos como prácticas sociales insertas en un contexto particular (Street 1995; Barton and Hamilton 1998).

En este marco, el presente estudio se centra especialmente en algunas de las prácticas relacionadas al desarrollo de la lectura y escritura. Debido a la relevancia social de la alfabetización, se plantea la necesidad de considerar de forma social la lectura y la escritura, puesto que esto 
involucra poner en la palestra factores que influyen en el desarrollo de la construcción del significado y la comprensión. Esta investigación reconoce el enorme impacto de la alfabetización en la vida cotidiana. Es debido a esta toma de conciencia que uno de los intereses de esta investigación es contribuir a la comprensión de la literacidad en Chile.

El contexto sociocultural de la sociedad chilena es un factor que ciertamente se ve involucrado en la enseñanza, el aprendizaje y desarrollo de la lectura y la escritura. Aún más, de este contexto surgen prácticas distintivas y diferenciadoras que enmarcan la literacidad en un contexto particular. En este sentido, un factor importante a considerar es que el lugar principal donde los niños pueden desarrollar sus habilidades de lectura y escritura es en la escuela. Este hecho se contrapone con la experiencia de los países desarrollados, donde en su mayoría, los expertos mencionan también al hogar como uno de los aspectos a considerar en el desarrollo de la lectura y la escritura (Heath 1983; Barton and Hamilton 1998). Esta perspectiva, sin embargo, se ve afectada por la disponibilidad de recursos económicos, por lo que en hogares de mayor vulnerabilidad las prácticas de lectura y escritura en el hogar representan grandes diferencias respecto a lo observado en los hogares de niños clase media. Las prácticas de literacidad familiar en un contexto de vulnerabilidad tienden a ser escasas, por lo que la literacidad temprana, o alejada del sistema educacional se vuelve muy difícil (Strasser and Lissi 2009).

Por otro lado, en general en los países no desarrollados, las prácticas de literacidad familiar se ven afectadas por la falta de tiempo que los padres pueden destinar a sus hijos y la inequidad de recursos disponibles en el hogar. Con respecto a los padres, se debe mencionar además que por lo general los niveles de alfabetización tienden a ser menores que los de países desarrollados. En Chile, esta tesis se ve reflejada en los índices de alfabetización funcional adulta (Bravo and Contreras 2001; Torres 2009; SITEAL 2010). Si bien Chile se ubica por sobre el promedio latinoamericano, los índices se ubican muy por debajo de los estándares de los países desarrollados. Este factor impacta profundamente en la calidad de la literacidad familiar y en las prácticas y actitudes de los padres hacia la lectura y la escritura(Strasser and Lissi 2009). En este sentido, la literacidad familiar cumple un rol bastante distinto al que puede observarse en los países desarrollados y por ende, es de gran importancia aportar a esta discusión. 


\section{Marco de referencia}

Uno de los primeros puntos a explicitar dentro de las referencias teóricas que guía este estudio es la discusión en torno al concepto de literacidad. El origen del concepto proviene de la palabra anglosajona $<$ literacy $>$. De acuerdo a una definición lexicográfica, este concepto es definido como la habilidad de leer y escribir (MerriamWebster dictionary, Oxford). Es a partir de estas referencias que el término ha sido traducido como alfabetización en español. Sin embargo, tal como David Barton explica en Literacy: an introduction to the ecology of written language (1994), la palabra <literacy> ha sufrido cambios en el tiempo y la definición lexicográfica ha sido paulatinamente desplazada por una concepción arraigada al discurso en torno a la lectura y la escritura.

De acuerdo a Barton, existen al menos dos discursos públicos en torno al concepto: un discurso relacionado a las habilidades asociadas a la lectura y la escritura y un discurso asociado con una perspectiva de elite respecto de la literatura y la cultura (Barton 1994). Ambos discursos han sido adoptados indistintamente por los medios de comunicación y los responsables de las políticas públicas asociadas a la lectura y la escritura. El discurso asociado a las habilidades expresa una visión continua de la lectura y la escritura, se considera que ambas se desarrollan en el tiempo y por ende, van incrementando su complejidad. Por otro lado, el discurso asociado a la elite implica que la lectura y la escritura son prácticas prestigiosas socialmente, y que por ende se engarzan con una visión elitista de la cultura.

En el actualidad, en el contexto anglosajón literacy es utilizado para referir a las habilidades asociadas a la lectura y la escritura, pero trascendiendo la decodificación y enfatizando la comprensión de los textos y la producción textual en general. En este contexto, el concepto expandió notoriamente su significado, lo que generó una dificultad al intentar traducir el término a otros idiomas.

En el mundo hispanohablante, el debate se ha centrado en la posibilidad de traducir el concepto a una palabra en idioma español. En este sentido se propone la palabra <literacidad> como un neologismo originado en el debate académico que intenta trasladar el significado de la palabra inglesa a una palabra en español (Cassany 2006). Sin embargo, la propuesta de Cassany presenta el uso de la palabra $<$ literacidad $>$ no 
solo como una traducción sino también como una manera de establecer una posición ideológica respecto de la lectura y la escritura. Por medio de este concepto, Cassany también establece la relevancia del rol de la lectura y la escritura en la vida cotidiana de las personas. El concepto de < literacidad > conlleva un punto de vista que engloba la lectura y la escritura dentro de las prácticas sociales y con ella arraiga estas "habilidades" a un contexto particular. En este sentido, la palabra $<$ literacidad> propone un significado distinto al expresado por el discurso de las habilidades. Engloba otros significados, sin embargo, no por ello no reconoce la importancia la importancia del desarrollo de la lectura y la escritura a lo largo del tiempo.

En este marco, es importante destacar que para los propósitos de esta investigación ambos discursos sobre la lectura y la lectura y la escritura serán considerados. La relevancia de considerar ambos discursos radica en la posibilidad de identificar los logros y dificultados de los estudiantes en su escritura a la vez que relacionar los mismos a sus creencias en torno a la literacidad.

Desde un punto de visto sociocultural, la escritura se inserta dentro de un contexto social que moldea las prácticas de escritura: Los enfoques socioculturales de la escritura rechazan la concepción de la escritura como meros actos materiales de inscripción, por el contrario, la escritura es percibida como una cadena producción, recepción y distribución de corto y largo plazo (Prior 2006). La escritura se convierte así en el centro de una red social y cognitiva inserta en un contexto.

Además, el texto se construye como un artefacto y por medio de un proceso que involucra al contexto. En este sentido, el texto puede ser considerado como una práctica social que involucra toda la complejidad del contexto y la identidad.

\section{Construcción del conocimiento}

En el marco de este estudio, la construcción del conocimiento o significado emerge como el marco lingüístico que permite estudiar un fenómeno complejo como lo es el texto escrito. Para los propósitos de este estudio, la construcción del conocimiento en el texto escrito será evaluada en relación con la manera en que los estudiantes construyen su mundo por medio de la escritura. En palabras de Barton (1994): “El enfoque 
aquí descansa en una perspectiva constructivista del lenguaje. Cuando estudiamos metáforas y pensamiento, se observa cómo el lenguaje juega un rol central en los modelos mentales a través de los cuales las personas construyen el mundo"(1994 [Traducción propia]).

Debido a este enfoque, la construcción del conocimiento o del significado en el texto escrito se traduce en una estrategia que permite analizar cómo los estudiantes construyen el conocimiento en sus textos. Sin embargo, la construcción del significado no será analizada de manera aislada, sino que como parte de un contexto social relacionado con la percepción de los estudiantes en torno a la lectura y la escritura.

En relación a los antecedentes lingüísticos que componen la construcción del conocimiento, este proceso será representado por algunas de las prácticas discursivas que construyen la macroestuctura de un texto (Van Dijk and Kintsch 1983). De acuerdo a Van Dijk y Kintsch, la macroestructura es un todo coherente materializado por una red de sentencias interconectadas. Al mismo tiempo, la macroestructura se relaciona también con estrategias específicas, lo que implica que este concepto involucra un comportamiento orientado hacia una meta (la de construir el texto), un objetivo intencionado y consciente (Van Dijk and Kintsch 1983). En este contexto, la construcción del conocimiento será considerada a partir del conjunto de estrategias discursivas utilizadas por los estudiantes para construir significado en sus textos.

Con el fin de analizar la escritura de los estudiantes algunas estrategias particulares del modelo de Van Dijk y Kintsch serán tomadas en consideración. En específico, este estudio se centra en la Coherencia y la Coherencia local, puesto que ambos conceptos son centrales a la hora de reconstruir la manera en que los estudiantes construyen un texto. La Coherencia y Coherencia local son parte de la red mayor de conocimiento que involucra la construcción del significado desde un punto de vista cognitivo. La Coherencia no es meramente una característica de los textos, sino que es el complejo resultado de una interacción entre semántica, pragmática y los principios lógicos que tanto el lector como el escritor activan al momento de construir un significado coherente en el texto (Van Dijk and Kintsch 1983; Concha and Paratore 2011).

Por otro lado, en un nivel micro la Coherencia Local a su vez se relaciona con la cohesión, puesto que este concepto reúne las estrategias 
gramaticales específicas que son utilizadas al construir un texto. De acuerdo a Halliday y Hassan (1976), los diferentes conectores crean la textura que caracteriza la cohesión. En específico, este estudio se centra en las conjunciones y cualquier otra estrategia utilizada por los niños para unir sus oraciones. Es importante recalcar en este punto que debido a que este estudio se enfoca en estudiantes de segundo y cuarto año básico, las estrategias que utilizan se encuentran aún en vías de desarrollo y por ende, la evaluación se enmarca en los conocimientos esperados de los estudiantes de acuerdo al nivel en el que se encuentran.

Resulta relevante establecer que en paralelo al marco teórico lingüístico en el que se basa este estudio se encuentra una perspectiva social del desarrollo cognitivo (Rogoff 1990). Desde esta perspectiva el desarrollo cognitivo de los infantes se entiende enmarcado en un proceso social donde los pares juegan un rol fundamental.

En este sentido, el aprendizaje se desarrolla en un contexto social determinado y con la ayuda de los pares y no solo de los profesores o los padres. Este enfoque resulta de vital importancia para este estudio, puesto que se pretende comprender cómo los niños se relacionan con su propio aprendizaje. Esta investigación se centra en particular en la lectura y la escritura, sin embargo estas prácticas se encuentran insertas en un contexto mayor de relaciones sociales y esfuerzos individuales (Rogoff 1990). Es por ello que esta investigación reconoce el valor no solo de la percepción de los estudiantes sino de los factores sociales que subyacen en la lectura y la escritura.

$\mathrm{Al}$ reconocer esta perspectiva los niños se convierten en agentes activos de su aprendizaje, por lo que adquieren un rol activo también dentro del proceso de aprendizaje. Este enfoque determina no solo la manera en que los niños aprenden, pero también pone énfasis en la relevancia de sus percepciones en torno su proceso de aprendizaje.

\section{Corpus y métodos}

El diseño de esta investigación consideró el uso de métodos cuantitativos y estrategias de análisis discursivo para el procesamiento de los datos.

La muestra de este estudio está compuesta por 107 estudiantes de una escuela municipal subvencionada ubicada en la sexta región, en 
Chile. Los alumnos cursan segundo $(\mathrm{N}=53)$ y cuarto $(\mathrm{N}=54)$ año básico y la muestra cuenta con 55 mujeres y 52 hombre. Los participantes se encuentran distribuidos en 2 cursos por nivel. Todos los participantes son chilenos y hablantes nativos de español. Los participantes fueron reclutados por muestra de conveniencia o no probabilística, lo que implica que la muestra fue seleccionada por conveniencia del investigador (Kiess 2002; McMillan and Schumacher 2008).

El colegio fue seleccionado a partir de un contacto establecido por la investigadora durante una evaluación educacional que el colegio desarrolló el año 2008 por primera vez y que repitió una vez al año hasta el año 2012. En este sentido, tanto las autoridades del colegio como el profesorado conocían de antemano a la investigadora y la investigadora conocía las dinámicas y particularidades del colegio.

Respecto de las características socioculturales del colegio, este se encuentra clasificado como un colegio urbano de clase media (MINEDUC). Esta clasificación implica que los padres tienen entre 11 y 12 años de escolaridad y declaran ingresos entre los 245.000 y 450.000 pesos mensuales. Además, alrededor del 55\% por ciento de los estudiantes se encuentran en condición de vulnerabilidad.

\section{Descripción del instrumento de evaluación}

Los estudiantes fueron invitados a responder una actividad tipo cuestionario durante su horario de clases. La evaluación fue planificada para una hora pedagógica (45 minutos) y el aula fue supervisada por un examinador capacitado previamente.

Las preguntas de selección múltiple fueron usadas para medir las percepciones y actitudes en relación a las prácticas de lectura en el hogar. Estas preguntas se enfocaron en cubrir los principales aspectos del desarrollo de la literacidad familiar en niños (Rodriguez, TamisLeMonda et al. 2009). Los aspectos considerados fueron los siguientes: Actividades de literacidad, recursos para el aprendizaje y relación con los pares o padres. Además, se incorporaron algunas preguntas específicas relacionadas a las percepciones y actitudes sobre la lectura. Estas preguntas apuntaban a la opinión de los estudiantes respecto de las prácticas de literacidad familiar. Cada aspecto fue medido con 2 preguntas, esta decisión se llevó a cabo debido a que la encuesta se enfoca 
primordialmente en explicitar una tendencia estadística y no busca ser exhaustiva.

Las respuestas a las preguntas se desarrollaron utilizando como base una escala Likert, sin embargo se realizó una adaptación de esta herramienta considerando la edad de los participantes en el estudio. De acuerdo a Chambers y Johnston, los niños tienden a tener opiniones extremas cuando son enfrentados a escalas de evaluación. Por ello, las respuestas intentaron cubrir de manera clara y en pocas opciones las posibles preferencias de los participantes.

La validez de estas preguntas se midió de acuerdo al contenido del test, lo implica que la validez se acumula a partir de la experiencia de expertos y los participantes de la muestra (McMillan and Schumacher 2008). Para este estudio, la validación de expertos fue dada a partir de la revisión de la literatura y la experiencia de la investigadora en evaluación educativa. La validación de los participantes se desarrolló a partir de un estudio piloto llevado a cabo con 10 estudiantes que cumplían con la edad para participar en la muestra pero que no participaron en el estudio definitivo.

La consistencia de las preguntas de selección múltiple se evaluó a través del coeficiente Alfa de Cronbach, el que arrojó un resultado de .629 , lo que sugiere un índice de confiabilidad moderada respecto del test (Black 1999). Si bien para este índice de fiabilidad se considera aceptable un valor superior a .7, debido a que esta evaluación posee menos de 10 ítems y la muestra es pequeña, el resultado indica un buen nivel de consistencia interna.

Tabla 1: Coeficiente de Cronbach de las preguntas de selección múltiple.

\section{Reliability Statistics}

\begin{tabular}{cccc}
\hline Alfa de Cronbach & $\begin{array}{l}\text { Alfa de Cronbach basado } \\
\text { en ítems estandarizados }\end{array}$ & N de Ítems & \\
\hline .629 & .640 & 8 \\
\hline
\end{tabular}


La pregunta de elicitación de escritura fue diseñada para analizar construcción del conocimiento en su escritura. Debido a la orientación teórica de este trabajo, esta pregunta fue desarrollada considerando una perspectiva social de la escritura, por lo que la instrucción fue dejada lo más abierta posible:

"Imagina que tienes que contarle algo sobre ti a una persona que no te conoce ¿qué le dirías? En el siguiente cuadro puedes escribir algo sobre ti que te gustaría contarle a alguien. Puede ser sobre algo que te guste, tu familia, tus amigos, tus vacaciones, tu colegio, etc."

Esta instrucción presupone que el estudiante tiene la posibilidad de construir una parte de mundo mediante la escritura (Barton 1994). La pregunta abierta buscó fomentar la libertad de escritura de los estudiantes con el fin de fomentar la creatividad de los alumnos a la vez que posibilita la transformación del conocimiento en el texto escrito. De acuerdo a Bereiter \& Scardamalia (1987) con estructuras más guiadas los niños tienden a descargar imágenes desde su memoria sin hacer una transformación del conocimiento, por lo que la libertad fue clave para desarrollar este estudio.

Por último, el espacio para contestar fue diseñado como un cuadro blanco sin líneas. Esta elección buscaba facilitar la creatividad de los estudiantes y permitirles utilizar el espacio de la manera que ellos estimaran conveniente. Por medio de lo anterior, se esperaba contribuir a la libertad de los estudiantes en cuanto a las estrategias de construcción del significado que desearan utilizar.

\section{Procedimientos de análisis}

Para efectuar el análisis del cuestionario las preguntas de selección múltiple y la pregunta de elicitación se utilizaron diferentes métodos de análisis.

Las preguntas de selección múltiple fueron analizadas asignando un valor numérico a cada una de las posibles respuestas. Esto permitió realizar estadísticas descriptivas de la muestra y analizar las preguntas en cuanto a frecuencias, correlaciones y algunas medidas que serán explicadas en la sección de resultados.

La pregunta de elicitación se analizó mediante rúbricas de evaluación que fueron diseñadas para este estudio. La elaboración de las rúbricas se 
realizó considerando que mediante estas escales el investigador puede elaborar una matrix de los criterios específicos que permiten el aprendizaje (Martinez - Rojas 2008). En este sentido, las rúbricas permitieron describir las características específicas de cada texto a la vez que evaluar algunas de las dificultades de los alumnos. Las rúbricas fueron desarrolladas de manera inductiva, lo que implica que luego de efectuar la muestra y hacer una revisión de los textos se procedió a identificar algunas características particulares que eran comunes y significativas para describir el conjunto de textos. Luego de esta revisión se identificaron algunos descriptores que identificaban cada una de las dimensiones evaluadas.

El proceso de construcción de las rúbricas incluyó además una revisión de la literatura (Van Dijk and Kintsch 1983; Concha and Paratore 2011; Sotomayor, Molina et al. 2013) y el currículo de Lenguaje y Comunicación y los mapas de progreso del aprendizaje (MINEDUC). De acuerdo a lo anterior y considerando las características de los textos, se definió analizar 4 dimensiones: Coherencia, Coherencia Local, Contenido y Estructura. Cada dimensión fue diseñada con 4 niveles de desempeño siendo 4 el de mejor desempeño. Estos niveles buscaban caracterizar el desempeño de los estudiantes en relación a lo esperado en el nivel y además caracterizar el texto en relación con el resto de la muestra. En este sentido, el nivel más alto de desempeño se desarrolló no solo pensando en el desempeño ejemplar, sino que también se fijó un estándar en relación a la muestra. A continuación se presenta una descripción de cada dimensión:

Coherencia: Para este estudio, la Coherencia del texto se focaliza principalmente en el propósito comunicativo y en el sentido global del texto. En este sentido, el nivel de desempeño se evaluó en relación al propósito comunicativo y su efectividad.

Coherencia Local: Esta dimensión se relaciona a la coherencia global, sin embargo, se focaliza en la ausencia, presencia y efectividad de las estrategias para conectar los distintos significados del texto.

Contenido: Esta dimensión pretende evaluar la respuesta del estudiante y su construcción del significado en relación con la pregunta del instrumento de evaluación.

Estructura: Esta dimensión evalúa la estructura del texto en el sentido de las diversas unidades que componen un texto. Se consideraron el uso de párrafos, oraciones y signos de puntuación como descriptores del desempeño de loes estudiantes respecto de cómo estructurar un texto escrito. 
Es importante mencionar que los niveles de desempeño de las rúbricas fueron adaptados para cada curso. La adaptación involucró la dificultad de los descriptores, sin embargo se utilizaron los mismos descriptores con el fin de hacer comparable la muestra. Estas rúbricas no incluyeron el cálculo de aspectos específicos o errores de ortografía para la asignación de los puntajes.

\section{Resultados}

Esta sección da cuenta de algunos de los hallazgos de este estudio en relación a cómo los niños construyen sus significados en el texto escrito y cuál es la relación de sus creencias en torno a la literacidad.

En cuanto a los resultados de las preguntas de selección múltiple, cabe mencionar que el objetivo de estas preguntas fue medir una tendencia por parte de los estudiantes en cuanto a sus percepciones y las prácticas que conllevan el desarrollo de la lectura y la escritura. Las preguntas cubren los aspectos más importantes relacionados a la lectura y la escritura tanto en el hogar como en la escuela.

En este punto es importante recordar que los resultados presentados a continuación deben ser analizados con precaución puesto que se trata de un muestreo no probabilístico. Por lo tanto, los resultados no pueden ser generalizables a otras poblaciones. Por último, cabe destacar que en este estudio se privilegió explicitar las creencias de los estudiantes respecto de las actividades y recursos, por lo que no se especificó por ejemplo la frecuencia de lo que implicaba la respuesta a veces. Por ello, cada estudiante expresó la respuesta desde su particular percepción del mundo.

La tabla número 2 muestra los resultados en términos de frecuencia de aparición por pregunta. Los porcentajes dan cuenta del número de estudiantes por nivel que respondió cada alternativa. 
Tabla 2: Resultados de las preguntas de selección múltiple.

\begin{tabular}{|c|c|c|c|c|c|}
\hline & Pregunta & Curso & No & A veces & $\mathrm{Si}$ \\
\hline \multirow{2}{*}{$\begin{array}{l}\text { Percepciones } \\
\text { sobre la lectura }\end{array}$} & \multirow{2}{*}{$\begin{array}{l}\text { ¿Te gustó aprender a } \\
\text { leer? }\end{array}$} & 2 & $1.9 \%$ & $0.0 \%$ & $98.1 \%$ \\
\hline & & 4 & $0.0 \%$ & $3.8 \%$ & $96.2 \%$ \\
\hline \multirow{2}{*}{$\begin{array}{l}\text { Percepciones } \\
\text { sobre la lectura }\end{array}$} & \multirow{2}{*}{$\begin{array}{l}\text { ¿Te gusta cuando } \\
\text { alguien te lee? }\end{array}$} & 2 & $24.1 \%$ & $13.0 \%$ & $63.0 \%$ \\
\hline & & 4 & $11.3 \%$ & $18.9 \%$ & $69.8 \%$ \\
\hline \multirow{2}{*}{ Actividades } & \multirow{2}{*}{$\begin{array}{l}\text { ¿Te gusta leer todo lo } \\
\text { que ves? }\end{array}$} & 2 & $7.4 \%$ & $14.8 \%$ & $77.8 \%$ \\
\hline & & 4 & $1.9 \%$ & $56.6 \%$ & $41.5 \%$ \\
\hline \multirow{3}{*}{ Actividades } & \multirow{3}{*}{$\begin{array}{l}\text { ¿Con que frecuencia } \\
\text { lees en tu casa? }\end{array}$} & & Nunca & A veces & Muy seguido \\
\hline & & 2 & $11.1 \%$ & $27.8 \%$ & $61.1 \%$ \\
\hline & & 4 & $0.0 \%$ & $54.7 \%$ & $45.3 \%$ \\
\hline \multirow{3}{*}{ Recursos } & \multirow{3}{*}{$\begin{array}{l}\text { ¿Cuántos libros hay } \\
\text { en tu casa? }\end{array}$} & & Ninguno & Unos pocos & Muchos \\
\hline & & 2 & $7.4 \%$ & $18.5 \%$ & $74.1 \%$ \\
\hline & & 4 & $0.0 \%$ & $13.2 \%$ & $86.8 \%$ \\
\hline \multirow{3}{*}{ Recursos } & \multirow{3}{*}{$\begin{array}{l}\text { ¿Te gustaria que } \\
\text { alguien te regalara un } \\
\text { libro para tu } \\
\text { cumpleaños? }\end{array}$} & & No & $\begin{array}{c}\text { Depende del } \\
\text { libro }\end{array}$ & Yes \\
\hline & & 2 & $13.0 \%$ & $24.1 \%$ & $63.0 \%$ \\
\hline & & 4 & $1.9 \%$ & $58.5 \%$ & $39.6 \%$ \\
\hline \multirow{3}{*}{$\begin{array}{l}\text { Participación } \\
\text { de los padres }\end{array}$} & \multirow{3}{*}{$\begin{array}{l}\text { ¿Aprendes nuevas } \\
\text { palabras en tu casa? }\end{array}$} & & Nunca & A veces & Muy seguido \\
\hline & & 2 & $0.0 \%$ & $31.5 \%$ & $68.5 \%$ \\
\hline & & 4 & $0.0 \%$ & $20.8 \%$ & $79.2 \%$ \\
\hline \multirow{3}{*}{$\begin{array}{l}\text { Participación } \\
\text { de los padres }\end{array}$} & \multirow{3}{*}{$\begin{array}{l}\text { ¿Hablas con tus } \\
\text { papás sobre tu día? }\end{array}$} & & $\begin{array}{c}\text { Solo los fines } \\
\text { de semana }\end{array}$ & $\begin{array}{c}\text { Algunos } \\
\text { días }\end{array}$ & Todos los días \\
\hline & & 2 & $11.1 \%$ & $29.6 \%$ & $59.3 \%$ \\
\hline & & 4 & $1.9 \%$ & $39.6 \%$ & $58.5 \%$ \\
\hline
\end{tabular}

En cuanto a las percepciones respecto de la lectura, existe una clara tendencia positiva. La gran mayoría de los estudiantes declara poseer actitudes positivas en torno a la lectura. Sin embargo, en la pregunta relacionada a las percepciones sobre la lectura en voz alta existe una mayor variabilidad en los resultados.

Respecto de las actividades de literacidad, los resultados muestran una mayor variabilidad. Los estudiantes de cuarto año mencionan que no desarrollan actividades asociadas a la lectura de manera diaria.

En relación a los recursos para el aprendizaje, la tendencia muestra que la mayoría de los estudiantes percibe que tiene muchos libros en su casa. La pregunta respecto de si les gustaría como regalo un libro muestra que los estudiantes de mayor edad poseen preferencias más marcadas respecto a sus preferencias de lectura.

Finalmente, la participación de los padres muestra que la mayoría de los estudiantes expresa que aprenden palabras nuevas en el hogar y hablan frecuentemente con sus padres sobre sus actividades cotidianas. 
En cuanto a las diferencias por grupo curso, es interesante mencionar que solo en algunas preguntas se presentan diferencias significativas entre cursos. Este es el caso de las pregunta número 5 y 7 para segundo año:

Tabla 3: Resultados por curso, pregunta 5.

\begin{tabular}{rrrr}
\hline \multirow{2}{*}{ Curso } & \multicolumn{3}{c}{ 5. ¿Cuántos libros hay en tu } \\
& \multicolumn{3}{c}{ casa? } \\
\cline { 2 - 4 } & Ninguno & $\begin{array}{c}\text { Unos } \\
\text { pocos }\end{array}$ & Muchos \\
\hline 2A & 2 & 4 & 31 \\
\hline 2B & 2 & 6 & 9 \\
\hline Total & 4 & 17 & 86 \\
\hline
\end{tabular}

Tabla 4: Resultados por curso, pregunta 7.

\begin{tabular}{|c|c|c|}
\hline \multirow{2}{*}{ Curso } & \multicolumn{2}{|c|}{$\begin{array}{l}\text { 7. ¿Aprendes nuevas palabras } \\
\text { cuando estás en tu casa? }\end{array}$} \\
\hline & A veces & Muy seguido \\
\hline $2 \mathrm{~A}$ & 5 & 32 \\
\hline $2 \mathrm{~B}$ & 12 & 5 \\
\hline Total & 28 & 79 \\
\hline
\end{tabular}


Tabla 5: Correlación de las variables.

\begin{tabular}{|c|c|c|c|c|c|c|c|c|c|c|c|c|c|c|c|}
\hline \multicolumn{16}{|c|}{ Correlaciones } \\
\hline & & Curso & Sexo & Q1 & $\mathrm{Q} 2$ & Q3 & O4 & Q5 & 06 & Q7 & Q8 & $\mathrm{Co}$ & CoLocal & Contenido & Estructura \\
\hline \multirow{2}{*}{ Curso } & Pearson Correlation & 1 & -105 & .002 & $.270^{-1}$ & .045 & .123 & $.192^{\circ}$ & -106 & .111 & .058 & .190 & $.422^{-1}$ & $.583^{\prime \prime}$ & $.463^{\prime \prime}$ \\
\hline & Sig. (2-tailed) & & 282 & .983 & .005 & .643 & 206 & .048 & 279 & .253 & .556 & .050 & .000 & .000 & .000 \\
\hline \multirow{2}{*}{ Sexo } & Pearson Correlation & -105 & 1 & .004 & .086 & .087 & $-247^{\circ}$ & .069 & $.203^{\circ}$ & -102 & .067 & -151 & -152 & $.283^{\prime \prime}$ & $-250^{\circ}$ \\
\hline & Sig. (2-tailed) & .282 & & .963 & .381 & 375 & .010 & .482 & .036 & .297 & .492 & .122 & .119 & .003 & .009 \\
\hline \multirow{2}{*}{ Q1 } & Pearson Correlation & .002 & .004 & 1 & $.288^{\circ}$ & .060 & .151 & . 164 & .112 & .178 & .071 & .145 & .081 & .002 & .024 \\
\hline & Sig. (2-tailed) & .983 & .963 & & .003 & 538 & .120 & .092 & .252 & .067 & .468 & .136 & .406 & .985 & .807 \\
\hline \multirow{2}{*}{ Q2 } & Pearson Correlation & $-270^{-1}$ & .086 & $288^{-1}$ & 1 & $.290^{\circ}$ & $.315^{-1}$ & 152 & $.333^{\prime \prime}$ & $271^{\prime \prime}$ & .133 & -115 & -130 & $-205^{\circ}$ & $.208^{\circ}$ \\
\hline & Sig. (2-tailed) & .005 & 381 & .003 & & .002 & .001 & .118 & .000 & .005 & .172 & .240 & .182 & .035 & .031 \\
\hline \multirow{2}{*}{ Q3 } & Pearson Correlation & -.045 & .087 & .060 & $290^{\circ}$ & 1 & $284^{\prime \prime}$ & .090 & $.311^{1}$ & .189 & -017 & .044 & .095 & -.028 & -149 \\
\hline & Sig. (2-tailed) & .643 & .375 & .538 & .002 & & .003 & .357 & .001 & .051 & .859 & .656 & .333 & .776 & .126 \\
\hline \multirow{2}{*}{ Q4 } & Pearson Correlation & .123 & $.247^{\circ}$ & .151 & $.315^{\circ}$ & $284^{-1}$ & 1 & .171 & .175 & $263^{-1}$ & .074 & .129 & .171 & .109 & .028 \\
\hline & Sig. (2-tailed) & 206 & .010 & .120 & .001 & .003 & & .079 & .072 & .006 & .450 & .184 & .079 & 262 & .775 \\
\hline \multirow{2}{*}{ Q5 } & Pearson Correlation & $.192^{\circ}$ & .069 & .164 & 152 & .090 & .171 & 1 & .029 & .104 & .093 & .018 & .000 & .050 & .012 \\
\hline & Sig. (2-tailed) & .048 & 482 & .092 & .118 & .357 & .079 & & .767 & 288 & .342 & 857 & 1.000 & 608 & .902 \\
\hline \multirow{2}{*}{ Q6 } & Pearson Correlation & -.106 & $.203^{\circ}$ & .112 & $.333^{-1}$ & $.311^{-1}$ & .175 & .029 & 1 & $314^{-1}$ & $275^{\prime \prime}$ & .052 & .060 & .098 & -117 \\
\hline & Sig. (2-tailed) & .279 & .036 & .252 & .000 & .001 & .072 & .767 & & .001 & .004 & .593 & .537 & .316 & .232 \\
\hline \multirow[b]{2}{*}{ Q7 } & Pearson Correlation & .111 & -.102 & .178 & $271^{\prime \prime}$ & .189 & $263^{\prime \prime}$ & .104 & $.314^{-1}$ & 1 & $229^{\circ}$ & -.107 & .000 & -.096 & -.030 \\
\hline & Sig. (2-tailed) & .253 & 297 & .067 & .005 & .051 & .006 & 288 & .001 & & .017 & .274 & 1.000 & .324 & .757 \\
\hline \multirow{2}{*}{ Q8 } & Pearson Correlation & .058 & .067 & .071 & .133 & .017 & .074 & .093 & $.275^{\circ}$ & $.229^{\circ}$ & 1 & .023 & -123 & .100 & .025 \\
\hline & Sig. (2-tailed) & .556 & .492 & .468 & 172 & .859 & .450 & .342 & .004 & .017 & & .811 & 207 & .303 & .800 \\
\hline \multirow{2}{*}{ Coherencia } & Pearson Correlation & .190 & -.151 & . 145 & -.115 & .044 & .129 & .018 & -.052 & -107 & -.023 & 1 & $.302^{\prime \prime}$ & $.498^{\prime \prime}$ & $.429^{\circ}$ \\
\hline & Sig. (2-tailed) & .050 & .122 & .136 & 240 & .656 & . 184 & .857 & .593 & .274 & .811 & & .002 & .000 & .000 \\
\hline \multirow{3}{*}{$\begin{array}{l}\text { Coherencia } \\
\text { Local }\end{array}$} & Pearson Correlation & $.422^{-1}$ & -152 & .081 & -130 & .095 & .171 & .000 & .060 & .000 & -123 & $.302^{-1}$ & 1 & $.482^{\prime \prime}$ & $.446^{\prime \prime}$ \\
\hline & Sig. (2-tailed) & .000 & .119 & .406 & 182 & 333 & .079 & 1.000 & .537 & 1.000 & 207 & .002 & & .000 & .000 \\
\hline & & 107 & 107 & 107 & 107 & 107 & 107 & 107 & 107 & 107 & 107 & 107 & 107 & 107 & 107 \\
\hline \multirow{2}{*}{ Contenido } & Pearson Correlation & $.583^{\prime \prime}$ & $.283^{\prime \prime}$ & .002 & $-205^{\circ}$ & -028 & .109 & .050 & .098 & .096 & .100 & $.498^{\circ}$ & $.482^{\circ}$ & 1 & $.565^{\prime \prime}$ \\
\hline & Sig. (2-tailed) & .000 & .003 & .985 & 035 & .776 & 262 & 608 & .316 & .324 & .303 & .000 & .000 & & .000 \\
\hline \multirow{2}{*}{ Estructura } & Pearson Correlation & $.463^{-1}$ & $.250^{-1}$ & .024 & $-208^{\circ}$ & -149 & .028 & .012 & -117 & .030 & .025 & $.429^{-1}$ & $.446^{-1}$ & $.565^{\circ}$ & 1 \\
\hline & Sig. (2-tailed) & .000 & .009 & .807 & .031 & .126 & .775 & .902 & .232 & .757 & .800 & .000 & .000 & .000 & \\
\hline
\end{tabular}

-. Correlation is significant at the 0.01 level (2-tailed).

$\cdot$ Correlation is sianificant at the 0.05 level (2-tailed).

Resulta particularmente interesante la diferencia en la pregunta número 7, puesto que la diferencia entre un curso y otro puede estar dada debido al trabajo de aula de la profesora. Es decir que la variabilidad del resultado en cuanto a esta práctica en el hogar se encuentra influida por una práctica específica de la profesora en la escuela. Los alumnos de cuarto año mostraron en general una variabilidad no significativa en cuanto a la diferencia por curso.

Por último, se midieron las correlaciones de las preguntas de selección múltiple. Los resultados muestran que la pregunta número 2 ( ¿Te gusta leer todo lo que ves?) correlaciona con todas las preguntas a excepción de la pregunta 5 (¿Cuántos libros hay en tu casa?). En este sentido, se valida la importancia de fomentar la lectura cotidiana y de variados tipos de texto, puesto que este indicador impacta en las percepciones respecto de la lectura y la escritura. 
Las correlaciones muestran que la edad de los estudiantes no es significativa a la hora de describir los resultados. Este resultado se complementó aplicando una correlación parcial para explorar la relación entre las dimensiones evaluadas y la edad de los estudiantes. Los resultados arrojaron que el tipo de pregunta y respuesta de los estudiantes no afectó la relación de las variables, por lo que se confirmó la ausencia de correlación significativa entre las creencias de los estudiantes sobre literacidad y la edad.

Cabe mencionar que este resultado también puede estar influenciado por el currículum de Lenguaje y Comunicación. El actual currículum explicita como uno de los objetivos del primer ciclo básico, que los estudiantes desarrollen actitudes positivas hacia la lectura (MINEDUC 2012). Debido a lo anterior, es muy probable que los profesores estén realizando actividades que fomenten este tipo de creencias. Este énfasis en desarrollar actitudes positivas puede haber influenciado a los estudiantes a la vez que niega la posibilidad de que se generen opiniones menos positivas.

Respecto de los resultados de la pregunta de escritura, cabe mencionar que el objectivo de esta pregunta apuntaba a elicitar la escritura de los estudiantes a partir de una pregunta abierta. Por medio de este ítem se abordó la construcción del significado en el texto escrito.

El análisis por medio de rubricas arrojó los siguientes resultados:

Tabla 6: Resultados por nivel, Pregunta de elicitación.

\begin{tabular}{lrrrrr}
\hline Dimensión & Curso & Nivel 1 & Nivel 2 & Nivel 3 & Nivel 4 \\
\hline Coherencia & 2 & $0.0 \%$ & $55.6 \%$ & $38.9 \%$ & $5,6 \%$ \\
\cline { 2 - 6 } & 4 & $1.9 \%$ & $37.7 \%$ & $43.4 \%$ & $7.0 \%$ \\
\hline Coherencia & 2 & $24.1 \%$ & $72.2 \%$ & $3.7 \%$ & $0.0 \%$ \\
\cline { 2 - 6 } Local & 4 & $0.0 \%$ & $79.2 \%$ & $20.8 \%$ & $0.0 \%$ \\
\hline \multirow{2}{*}{ Contenido } & 2 & $7.4 \%$ & $42.6 \%$ & $38.9 \%$ & $11.1 \%$ \\
\cline { 2 - 6 } & 4 & $0.0 \%$ & $1.9 \%$ & $47.2 \%$ & $50.9 \%$ \\
\hline \multirow{2}{*}{ Estructura } & 2 & $27.8 \%$ & $51.9 \%$ & $14.8 \%$ & $5.6 \%$ \\
\cline { 2 - 6 } & 4 & $0.0 \%$ & $43.4 \%$ & $34.0 \%$ & $22.6 \%$ \\
\hline
\end{tabular}


En cuanto a la distribución de la muestra, los resultados muestran una distribución normal a excepción de la dimensión de Contenido, en donde la distribución se encuentra ligeramente inclinada hacia los niveles 3 y 4 . Sin embargo, cabe destacar que la dimensión de Coherencia Local agrupa la mayor cantidad de alumnos en el nivel 2 de logro. Este nivel de desempeño indica que los estudiantes poseen dificultades para utilizar recursos de la cohesión o los utilizan con muy poca frecuencia. En cuanto a las diferencias por grupo curso y dimensión, los resultados no muestra mayores diferencias por grupo. En general, los estudiantes muestran una distribución normal en ambos cursos por nivel.

Respecto de la correlación de las variables en la pregunta de escritura, cabe destacar que la Coherencia correlaciona con todas las otras variables, por lo que se pone de manifiesto la relevancia de esta dimensión en la escritura en general (ver tabla número 5). Es interesante destacar además que la edad es un factor que correlaciona con la Coherencia, la Coherencia Local y la Estructura. Esta relación indica que el factor edad posee una relación lineal directa perfecta con la manera en que los estudiantes se desempeñan en la escritura. En este sentido, puede especularse que estas dimensiones son acumulativas en el tiempo.

\section{Relación de la pregunta de escritura y las preguntas de selección múltiple}

Para evaluar la relación de la escritura de los estudiantes con sus percepciones sobre la literacidad se efectuaron análisis estadísticos que relacionaron los resultados de las preguntas de selección múltiple con los resultados de la pregunta abierta. Este análisis se efectuó para determinar la relación que existe entre las creencias sobre literacidad y los recursos de construcción del significado en el texto escrito.

Para establecer esta relación, se aplicaron correlaciones y además, debido a las características de la muestra y las variables se aplicó el Test de Kruskal Wallis. Estos resultados arrojaron que no existe una correlación estadísticamente significativa entre los resultados de la pregunta de selección múltiple y el desempeño de los estudiantes en el texto escrito.

Por último, se efectuó un análisis considerando las variables de edad y género. Los resultados mostraron que las diferencias por género son más significativas que las diferencias por edad. Las alumnas mostraron 
mejores resultados en su desempeño en las dimensiones evaluadas en la escritura y una mayor homogeneidad en cuanto a las tendencias positivas respecto de sus creencias sobre la literacidad. Estos resultados se relacionan a los hallazgos de investigaciones internacionales anteriores que muestran un mejor desempeño del género femenino en las habilidades relacionadas a la lectura y la escritura ${ }^{2}$.

Esta diferencia de género resulta aún más interesante a la luz de esta investigación, puesto que se consideraron estudiantes de entre 7 y 10 años, pero aun así la diferencia de género resultó estadísticamente más significativa. Estos resultados podrían indicar que la diferencia de género se mantiene a través del tiempo, sin embargo, requiere de mayor investigación en el área para apoyar esta hipótesis.

\section{Conclusión y alcance del estudio}

En relación con las preguntas de selección múltiple, aun cuando modesta, este estudio entrega evidencia en torno a las percepciones y actitudes hacia la literacidad. En este sentido, los resultados ponen de manifiesto una clara tendencia positiva entre los estudiantes participantes en el estudio. Aun cuando este resultado puede ser fundamentado en el currículum, estos resultados pueden ser considerados como un buen indicador para la instalación de futuras estrategias que impacten positivamente en la lectura y la escritura.

Sin embargo, cabe mencionar que los estudiantes manifestaron una mayor variabilidad al expresar la frecuencia con que realizan las actividades asociadas a la lectura y la escritura. En este sentido, resulta relevante destacar que estos estudiantes están ciertamente expuestos diariamente a distintos recursos de lectura y escritura tales como: avisos publicitarios, revistas, panfletos, textos en la televisión, tareas escolares y todas las actividades asociadas a la escuela etc. Consecuentemente, resulta un tanto difícil que los estudiantes desempeñen actividades asociadas a la lectura y la escritura con una baja frecuencia.

2 Investigaciones anteriores incluyen el informe del National Literacy Trust en Inglaterra (Boys' Reading Commision, 2012), en Estados Unidos la evaluación Nacional de Progreso educativo mostró resultados similares en 2009 y 2011 (US National Assessment of Educational Progress, NAEP, 2009 - 2011) y por último, el Ministerio de Educación de Ontario, Canadá encargó un informe para incrementar los resultados de los varones en cuanto a sus habilidades de lectura y escritura (Me read? No way! A practical guide to improving boys' literacy skills, 2004). 
En este sentido, este estudio muestra que los estudiantes perciben que no realizan actividades frecuentemente, aun cuando es muy poco probable que así sea. Es por ello, que esta investigación también pone el foco en el concepto de lectura y escritura que poseen los estudiantes. Mediante una ampliación del concepto (hacia un enfoque más sociocultural de la lectura y la escritura), los alumnos podrían reconocer y validar instancias de aprendizaje distintas a las que están acostumbrados a trabajar en el colegio.

Respecto de los recursos, el hecho de que la gran mayoría de los estudiantes expresó tener muchos libros en sus hogares da cuenta de un punto interesante de abordar en futuras investigaciones. Este resultado resulta interesante puesto que contradice la percepción popular y estudios anteriores que mencionan los pocos recursos que existirían en los hogares chileno (Susperreguy, Strasser et al. 2007; Strasser and Lissi 2009). Debido a las características del estudio, esta tendencia puede indicar que los estudiantes efectivamente poseen una gran cantidad de libros o no. Sin embargo, resulta interesante destacar que los estudiantes en su mayoría perciben que tienen los recursos necesarios para desarrollar su lectura y escritura, lo que ya genera una posición positiva para generar nuevas estrategias que los ayuden a mejorar estas habilidades. En este sentido, esta conciencia puede aprovecharse por medio de la creación de oportunidades para que los alumnos trabajen, tanto en el hogar como en la escuela, con estos recursos.

En relación con la pregunta de escritura, los resultados identificaron algunos aspectos específicos de la escritura en los cuales los aprendizajes se encuentran disminuidos. En este punto, la dimensión de Coherencia Local mostró ser la que generó mayores problemas a los estudiantes. En este sentido, esta investigación dio cuenta de algunos de los problemas específicos de la escritura de los estudiantes, pero a la vez dio cuenta del valor de permitir libertad a los estudiantes en cuanto a su escritura. Aun cuando se identificaron problemas en cuanto a la puesta en práctica de algunas estrategias de escritura, los textos fueron capaces de construir significado con estrategias diferentes a las normalmente evaluadas. Las estrategias utilizadas por los estudiantes contribuyeron a la construcción de la coherencia del texto.

En cuanto a los resultados que evaluaron la relación entre las creencias de literacidad y la muestra de escritura, este estudio reveló 
que las prácticas y creencias en torno a la lectura y escritura en el hogar no son estadísticamente significativas respecto de los resultados en la producción textual. Si bien estos resultados pueden estar relacionados a las especificaciones del currículo, aún presentan interesante evidencia en torno a la relación de ambas variables para futuras investigaciones.

Además, los resultados mostraron que la variable de género tiene un mayor impacto que la de edad en cuanto a la riqueza de las estrategias de construcción de significado. En este sentido, el género de los estudiantes se presenta como un factor a considerar a la hora de desarrollar estrategias de aula que puedan contribuir a mejorar la escritura de los estudiantes.

Finalmente, este estudio propone reflexionar respecto del impacto de las prácticas de literacidad familiar en la producción textual. Y aun cuando los resultados presentados mostraron evidencia interesante al respecto, una de las limitaciones de este estudio fue que los alumnos posiblemente están fuertemente influenciados con el currículo. Por ello, futuras etapas de esta investigación contemplarán un estudio cualitativo que evalúe esta hipótesis. 


\section{Bibliografía}

Barton, D. (1994). Literacy: an introduction to the ecology of written language. Malden, Mass, Blackwell Pub.

Barton, D. and M. Hamilton (1998). Local literacies. Reading and writing in context. London, New York, Routledge.

Bereiter, C. and M. Scardamalia (1987). The psychology of written composition. Hillsdale, NJ, Lawrence Erlbaum.

Black, T. R. (1999). Doing quantitative research in the social sciences: an integrated approach to research design, measurement and statistics. London, SAGE.

Bravo, D. and D. Contreras (2001). Competencias y destrezas básicas de la población adulta en Chile. Santiago, Andros Impresores.

Cassany, D. (2006). Tras las líneas. Sobre la lectura contemporánea. Barcelona, Anagrama.

Chambers, C. T. and C. Johnston (2002). "Developmental Differences in Children's Use of Rating Scales." Journal of Pediatric Psychology 27(1): 27-36.

Concha, S. and J. Paratore (2011). "Local Coherence in Persuasive Writing: An exploration of Chilean student's metalinguistic knowledge, writing process, and writing product." Written Communication 28(1): 34-49.

Crespo, N., R. Benítez, et al. (2007). "La comprensión oral del lenguaje no literal y su relación con la producción escrita en escolares". Revista Signos 40(63): 31-50.

Figari, R. B. (2009). "Análisis descriptivo de narraciones escritas por niños y niñas de tercer año básico." Literatura y Lingüística 20: 103-123.

Halliday, M. A. K. and R. Hasan (1976). Cohesion in English. London, Longman.

Heath, S. B. (1983). Ways with words: language, life, and work in communities and classrooms. Cambridge Cambridgeshire; New York, Cambridge University Press. 
Kiess, H. (2002). Statistical concepts for the behavioural sciences. Boston, Allyn \& Bacon.

Martinez - Rojas, J. (2008). "Las rúbricas de evaluación escolar: Su construcción y su uso." Avances en Medición 6: 129138.

McMillan, J. H. and S. Schumacher (2008). Investigación educativa. Madrid, Pearson.

MINEDUC. (2008). Mapas de Progreso del Aprendizaje. Sector Lenguaje y Comunicación. Santiago, Chile, Ministerio de Educación, Gobierno de Chile.

MINEDUC. (2012). Bases Curriculares. Lenguaje y Comunicación: Educación Básica. Ministerio de Educación, Gobierno de Chile.

Prior, P. (2006). A sociocultural theory of writing. Handbook of writing research. C. A. MacArthur, S. Graham and J. Fitzgerald. New York; London, Guilford.

Rodriguez, E. T., C. S. Tamis-LeMonda, et al. (2009). "The formative role of home literacy experiences across the first three years of life in children from low-income families." Journal of Applied Developmental Psychology 30: 677694.

Rogoff, B. (1990). Apprenticeship in thinking. New York, Oxford University Press.

SITEAL (2010). El analfabetismo en América Latina, una deuda social. Sistema de información de tendencias educativas en América Latina

Sotomayor, C., D. Molina, et al. (2013). "Caracterización de problemas ortográficos recurrentes en alumnos de escuelas municipales chilenas de $3^{\circ}, 5^{\circ}$ y $7^{\circ}$ básico". Revista Signos 46(81): 105-131.

Strasser, K. and M. L. Lissi (2009). "Home and Instruction Effects on Emergent Literacy in a Sample of Chilean Kindergarten Children." Scientific studies of reading 13(2): 175-204. 
Street, B. V. (1995). Social literacies: critical approaches to literacy in development, ethnography, and education. London; New York, Longman.

Susperreguy, M. I., K. Strasser, et al. (2007). "Creencias y prácticas de literacidad en familias chilenas con distintos niveles educativos [Literacy beliefs and practices in Chilean families with different educational levels]." Revista Latinoamericana de Psicología 39(2): 239.

Torres, R. M. (2009). De la alfabetización al aprendizaje a lo largo de toda la vida: Tendencias, temas y desafíos de la educación de personas jóvenes y adultas en América Latina y el Caribe. Sintesis del reporte regional. Hamburgo, Alemania, UNESCO.

Van Dijk, T. A. and W. Kintsch (1983). Strategies ofdiscourse comprehension. New York, Academic Press. 